\title{
Large-Scale Computations Leading to a First- Principles Approach to Nuclear Structure
}

\author{
W. E. Ormand, P. Navratil
}

This article was submitted to Computational and Group Theoretical Methods in Nuclear Physics, Playa del Carmen, Mexico 02/18/2003 - 02/21/2003

U.S. Department of Energy

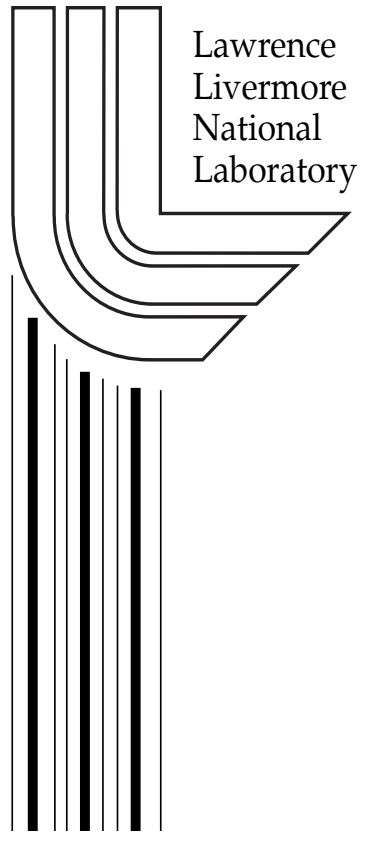

\section{August 18, 2003}


This document was prepared as an account of work sponsored by an agency of the United States Government. Neither the United States Government nor the University of California nor any of their employees, makes any warranty, express or implied, or assumes any legal liability or responsibility for the accuracy, completeness, or usefulness of any information, apparatus, product, or process disclosed, or represents that its use would not infringe privately owned rights. Reference herein to any specific commercial product, process, or service by trade name, trademark, manufacturer, or otherwise, does not necessarily constitute or imply its endorsement, recommendation, or favoring by the United States Government or the University of California. The views and opinions of authors expressed herein do not necessarily state or reflect those of the United States Government or the University of California, and shall not be used for advertising or product endorsement purposes. 


\title{
LARGE-SCALE COMPUTATIONS LEADING TO A FIRST-PRINCIPLES APPROACH TO NUCLEAR STRUCTURE
}

\author{
W. E. ORMAND AND P. NAVRÁTIL \\ Lawrence Livermore National Laboratory, \\ P.O. Box 808, L-414, \\ Livermore, $C A 94551$ \\ E-mail: ormand1@llnl.gov
}

\begin{abstract}
We report on large-scale applications of the $a b$ initio, no-core shell model with the primary goal of achieving an accurate description of nuclear structure from the fundamental inter-nucleon interactions. In particular, we show that realistic two-nucleon interactions are inadequate to describe the low-lying structure of ${ }^{10} \mathrm{~B}$, and that realistic three-nucleon interactions are essential.
\end{abstract}

\section{Introduction}

An important goal in the study of nuclear structure is to answer the question: Do we really understand how nuclei are put together? Towards this end, we wish to formulate a complete description of the properties of complex nuclei from first principles. In particular, we wish to determine if our knowledge of the fundamental interaction between pairs of nucleons is sufficient to describe the rich and complex structure observed in nuclei. This is an extremely difficult enterprise, and has really only been accomplished for the lightest of nuclei. Thus far, Faddeev-like ${ }^{1,2}$ approaches and the hyperspherical formalism ${ }^{3,4}$ have been applied to three- and four-body systems, while Monte Carlo methods ${ }^{5,6}$ have now been applied to systems with up to ten nucleons ${ }^{7}$. Also, the coupled-cluster expansion method $^{8}$ has been applied to ${ }^{16} \mathrm{O}$. Here, we utilized new developments in many-body theory and the exceptional computational power of the ASCI system at LLNL to perform a study of nuclear structure from first principles, i.e., an ab initio approach, for nuclei throughout the $p$-shell. 


\section{Effective interactions and the shell model}

The basic task at hand is to obtain solutions to the standard eigenvalue problem

$$
\left(\hat{H}-E_{\nu}\right) \Psi_{\nu}=0,
$$

where $E_{\nu}$ is the desired eigenvalue, $\hat{H}$ is the Hamiltonian, and $\Psi_{\nu}$ is the eigenfunction. One starting point for solving Eq. (1) is the interacting shell-model $^{9}$, where we introduce a set of orthogonal basis states $\phi_{i}$ to construct the exact solution, i.e., $\Psi_{\nu}=\sum_{i} c_{\nu i} \phi_{i}$. Solutions to Eq. (1) can then be obtained from a set of coupled equations that can be solved using matrix diagonalization techniques. The primary difficulty encountered is that because of the short-range repulsion in the nucleon-nucleon interaction, a basis of infinite dimension is required.

This infinite basis problem can, in principle, be circumvented by the use of effective-interaction theory. First, one chooses manageable subset of the original basis states, which is defined by the operator $\hat{P}$, leading to the slightly different eigenvalue problem

$$
\left(\hat{H}_{e f f}-E_{\nu}\right) \hat{P} \Psi_{\nu}=0,
$$

where $\hat{P} \Psi_{\nu}$ is the projection of the exact solution onto the chosen model space, $E_{\nu}$ is again the eigenvalue, and $\hat{H}_{e f f}$ is an effective Hamiltonian that yields the exact solution of Eq. (1). The excluded space is then usually defined by the operator $\hat{Q}$, with $\hat{P}+\hat{Q}=1, \hat{P}^{2}=\hat{P}, \hat{Q}^{2}=\hat{Q}$, and $\hat{P} \hat{Q}=\hat{Q} \hat{P}=0$.

An important feature of $\hat{H}_{e f f}$ is that it is composed of two-, three-, ..., $n$-body components even if the fundamental interaction is only pair-wise. The power of $H_{e f f}$ is that it may provide a mechanism to carry out computationally tractable calculations while including the relevant physics. For most potentials, the dominant correlations in the effective interactions are at the two-body level, but for smaller $P$-space, the higher-body correlations are essential for a correct result.

Here, we utilized a unitary transformation due to Lee and Suzuki ${ }^{10}$ to derive the effective interaction. This formalism is the foundation for the highly successful no-core shell model (NCSM) ${ }^{11,12}$. The procedure is based on finding the transformation, $e^{S}$, to the Hamiltonian so that the $P$ - and $Q$-spaces for the many-body problem are decoupled, i.e.,

$$
\hat{Q} e^{-S} \hat{H} e^{S} \hat{P}=0 .
$$

Strictly speaking, in this form, $\hat{H}_{\text {eff }}$ is not unitary, but can be made so. Explicit formulae for the $n$-body matrix elements are given by Eqs. (9) and 
(10) in Ref. ${ }^{12}$. Two important features are evident. First, in the limit that $\hat{P} \rightarrow 1$, the effective interaction tends to the bare interaction. Second, a subset of exact $n$-body solutions are required to determine the $\hat{H}_{\text {eff }}^{(n)}$. These exact solutions may be obtained by any method, e.g., large-basis shell-model calculations utilizing either the bare interaction or an $(n-1)$-body effective interaction.

Our calculations begin with a two-body (also plus three-body) Hamiltonian for the $A$-nucleon system, which depends on the intrinsic coordinates alone. We utilize realistic interaction potentials that are derived from nucleon-nucleon scattering data. To facilitate our calculations, we introduce an $A$-nucleon harmonic-oscillator Hamiltonian acting only on the center-of-mass, whose effect is subtracted from the many-body calculation. The primary advantages of the harmonic oscillator are that it acts as pseudo mean field providing a convenient basis for expanding the many-body wave function and that the relative motion of the center-of-mass can be separated from the intrinsic degrees of freedom exactly. Within the harmonicoscillator basis, we specify the $P$-space, designated by the maximum number, $N_{\text {max }}$, of oscillator quanta excitations, and construct the $A$-body basis. We then obtain the eigenvalues, $E_{\nu}$, using a shell-model code. This amounts to diagonalizing a symmetric matrix, whose dimensions are given by the number of $A$-body basis states. Although the dimensions can be quite large, efficient numerical techniques, such as Lanczos ${ }^{13}$, exist that yield the lowest eigenvalues. The parameters governing our convergence are then: $N_{m a x}$, defining the model-space; $n$, the number of clusters in the effective interaction; and $b=\sqrt{m \Omega / \hbar}$, the oscillator parameter setting the physical scale. Ideally, once convergence is achieved, the NCSM solution is independent of these parameters. In practice, however, our best solution is obtained for the largest $N_{\max }$ that is computationally feasible and a value of the oscillator parameter where the binding energy is least sensitive.

In general, computational limitations impose a compromise in the choice of $N_{\max }$ and $\hat{H}_{e f f}^{(n)}$. This is due to the fact that for each increment in $N_{\max }$ the number of $A$-body states increases dramatically. While for larger $n$, the number of interaction matrix elements increases and the sparsity of matrix decreases. In addition, the effective interaction itself is more difficult to evaluate for increasing $n$ and/or $N_{\max }$. To illustrate the level complexity of the three-body calculations, for $N_{\max }=4,39,523,066$ 3-particle interaction matrix elements are needed. In this space, the number of $M$-scheme 10body configurations for ${ }^{10} \mathrm{~B}$ with $J_{z}^{\pi}=0^{+}$is 581,740 , and the resultant matrix to be diagonalized has over $2.2 \times 10^{9}$ non-zero elements. 




Figure 1. Comparison of the NCSM and GFMC spectra obtained for the Argonne AV8' potential. The NCSM spectra are shown as a function of the model size denoted by $N_{\max } \hbar \Omega$.

\section{Nuclear Structure Calculations}

Over the past few years, several extensive studies have been performed with the NCSM using realistic NN-interactions such as the Argonne AV8 potentials ${ }^{6}$ and CD-Bonn ${ }^{14}$. These include first the $a b$ initio applications ${ }^{15}$ for ${ }^{12} \mathrm{C}$, an extensive study of $A=6$ nuclei $^{16}$, an examination of the nature of excited states in ${ }^{8} \mathrm{Be}$, and a recent large-basis application for $A=10$ nuclei $^{18}$. The study with $A=6$ provides an excellent example of the convergence and the utility of the no-core shell model ${ }^{16}$. In particular, in Fig. 1 , we compare the NCSM spectrum for ${ }^{6} \mathrm{Li}$ (as a function of the model space $N_{\max }$ ) using the Argonne AV8' potential with results obtained from the GFMC method. Overall, there is good agreement between the two methods. Also, as will be shown below, the converged NCSM value for the total binding energy ${ }^{6} \mathrm{Li}$ is agreement to within $400 \mathrm{keV}$ of the GFMC calculation.

The inclusion of higher-body clusters generally improves the overall convergence ${ }^{19}$. The Binding energies of the nuclei ${ }^{6} \mathrm{Li},{ }^{8} \mathrm{Be}$, and ${ }^{10} \mathrm{~B}$ are shown in Fig. 2. On the left-side of the figure the binding energies are plotted as a function of the oscillator parameter, which effectively defines the 

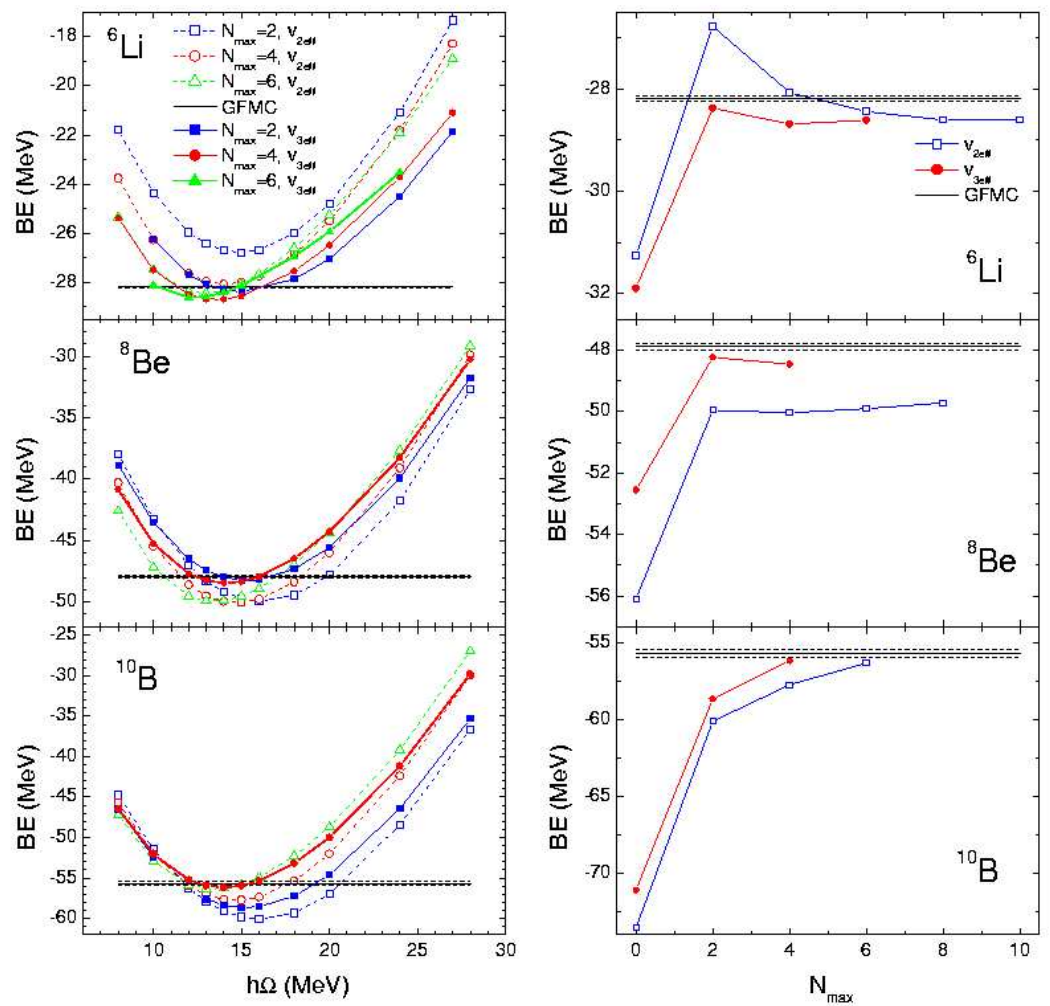

Figure 2. Calculated ground-state energy of ${ }^{6} \mathrm{Li}$ (upper panel), ${ }^{8} \mathrm{Be}$ (middle panel) and ${ }^{10} \mathrm{~B}$ (lower panel) using the $\mathrm{AV} 8^{\prime} \mathrm{NN}$ potential with Coulomb. Results using the twobody effective interaction and the three-body effective interaction in basis spaces up to $6 \hbar \Omega$ in the range of $\mathrm{HO}$ frequencies of $\hbar \Omega=8-28 \mathrm{MeV}$ are shown and compared to the GFMC results from Ref. ${ }^{6}$. On the rhs, the energies at the HO frequency minima as a function of $N_{\max }$ are plotted.

size of the nucleus. The figure shows parabolas for the various model spaces, which are denoted by the $N_{\max }$ value, and with two-body $\left(V_{2 e f f}\right.$ - dotted lines) and three-body ( $V_{3 e f f}$ - solid lines) effective interactions. In general, the behavior on the oscillator parameter is lessened (flatter parabola) as either the model space size increases or when more clusters are included in the effective interaction. The "best" result for a given model space is chosen in the region exhibiting the least dependence on the oscillator parameter. These "best" values are then plotted on the right-side of the figure as a function of the model space $N_{\max }$ and compared with the results from the 
GFMC method (full solid lines with a dotted line band denoting the GFM uncertainty). In general, for any given value of $N_{\max }$, faster convergence is achieved with higher clusters included in $H_{\text {eff }}$. In addition, we note that the NCSM calculation with the two-body effective interaction still differs from the GFMC result by $\approx 1.8 \mathrm{MeV}$ even for the largest model space. On the other hand, the three-body effective interaction results are in better agreement for smaller model spaces. Given that ${ }^{8} \mathrm{Be}$ is actually unbound, and is two alpha-particle resonance, this suggests that the three-body effective interactions includes more correlations into the wave function. Overall, the results obtained with the three-body clusters in the effective interaction are in agreement with the GFMC calculations to within $400 \mathrm{keV}$.

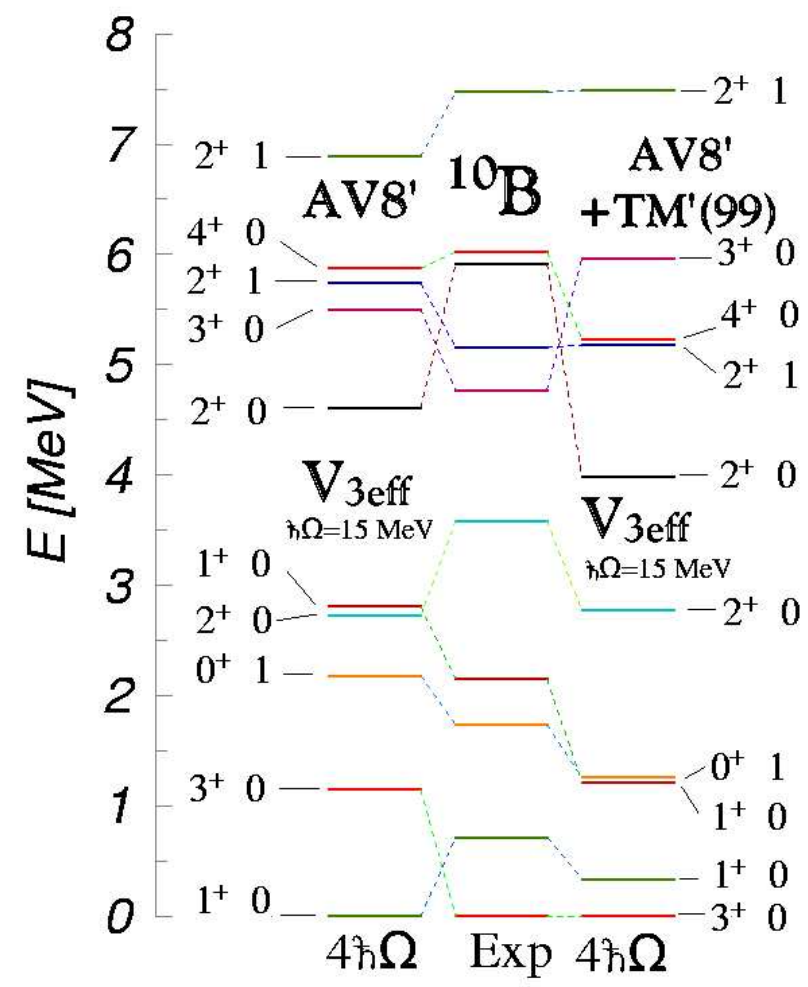

Figure 3. Comparison of low-lying spectrum of ${ }^{10} \mathrm{~B}$ obtained with the $\mathrm{AV} 8^{\prime}$ two-nucleon interaction alone(left side) and with the Tucson-Melbourne three-nucleon force (right side)with experiment. 
With confidence in convergence, we now turn to a more systematic study of the structure of light nuclei. A particularly salient example is ${ }^{10} \mathrm{~B}$. We show the specturn obtained with the AV8' in Fig. 3 (using a threebody effective interaction, $\left.V_{3 e f f}\right)$ in comparison with experiment. The most striking feature is that the ground state $\left(3^{+}\right)$and the first excited state $\left(1^{+}\right)$ are reversed in order. We must now conclude that realistic two-nucleon forces fail to describe the low-lying structure of ${ }^{10} \mathrm{~B}$. Indeed, this is a feature that appears to be common to all the realistic nucleon-nucleon forces. This is the first direct evidence that, in addition to providing extra binding, three-nucleon forces also impact nuclear structure.

We are then forced to conclude that a proper description of nuclear structure must a include so-called "true" three-nucleon force. Note that these are quite different from the three-body clusters that we included in the effective interaction, as these term are actually induced because of the effect of the finite model space. We have recently carried out calculations including the Tucson-Melbourne three nucleon force ${ }^{20}$ for ${ }^{10} \mathrm{~B}$. The results are shown in Fig. 3, where better agreement with the experimental spectrum is obtained, In particular, the ordering of the first two states is now correct. Overall, one finds that the three-nucleon interactions has spin-orbit components that play an important role in determining the structure of nuclei in the region $10 \leq A \leq 15$. At this stage, investigations are underway to determine if the remaining disagreement in Fig. 3 is due to: i) convergence of the three-body effective interaction, ii) the many-body model space, and iii) the form of the three-nucleon interaction itself.

\section{Conclusions}

Substantial progress has been made towards an exact description of nuclear structure. In this work, we describe the $a b$ initio, no-core shell model and recent results. In particular, we find that realistic NN interactions by themselves are inadequate and that three-nucleon forces play an important role in determining nuclear properties. Further research is currently underway.

\section{Acknowledgements}

This work was performed under the auspices of the U.S. Department of Energy by the University of California, Lawrence Livermore National Laboratory, under contract No. W-7405-Eng-48. This project received support through a Laboratory Directed Research and Development grant, tracking No. 00-ERD-028. 


\section{References}

1. L.D. Faddeev, Zh. Éksp. Teor. Fiz. 39, 1459 (1960) [Sov. Phys. JETP 12, 1014 (1961)]; O.A. Yakubovsky, Yad. Fiz. 5, 1312 (1966) [Sov. J. Nucl. Phys. 5, 937 (1967)].

2. H. Kamada and W. Glöckle, Nucl. Phys. A548, 205 (1992); J.L. Friar, G.L. Payne, V.G.J. Stoks, and J.J. de Swart, Phys. Lett. B 311, 4 (1993); H. Witala, D. Hüber, and W. Glökle, Phys. Rev. C49, R14 (1994); A. Nogga, D. Hüber, H. Kamada, and W. Glökle, Phys. Lett. B 409, 19 (1997); W. Glökle and H. Kamada, Phys. Rev. Lett. 71, 971 (1993).

3. M. Viviani, A. Kievsky, and S. Rosati, Few-Body Syst. 18, 25 (1995).

4. N. Barnea, W. Leidermann, and G. Orlandini, Nucl. Phys. A650, 427 (1999).

5. J. Carlson, Phys. Rev. C36, 2026 (1987); C38, 1879 (1988); J. Carlson and R. Schiavilla, in Few-Body Systems, suppl. 7, Ed. by B.L.G. Bakker and R. van Dantzig (Springer-Verlag, 1994) p. 349.

6. B.S. Pudliner et al, Phys. Rev C56, 1720 (1997); R.B. Wiringa, Nucl. Phys. A631, 70c (1998); R.B. Wiringa, S.C. Pieper, J. Carlson, and V.R. Pandharipande, Phys. Rev. C62, 014001 (2000); S.C. Pieper, V.R. Pandharipande, R.B. Wiringa, and J. Carlson, Phys. Rev. C64, 014001 (2001).

7. S.C. Pieper, private communication.

8. J.H. Heisenberg and B. Mihaila, Phys. Rev. C59, 1440 (1999); B. Mihaila and J.H. Heisenberg, Phys. Rev. C61, 054309 (2000); B. Mihaila and J.H. Heisenberg, Phys. Rev. Lett. 84, 1403 (2000).

9. P.J. Brussaard and P.W.M. Glaudemans, Shell-model applications in nuclear spectroscopy, (North-Holland, Amsterdam, 1977); R.D. Lawson, Theory of the nuclear shell model, (Clarendon Press, Oxford, 1980).

10. K. Suzuki and S.Y. Lee, Prog. Theor. Phys. 64, 2091 (1980); K. Suzuki, Prog. Theor. Phys. 68, 246 (1982).

11. P. Navrátil and B.R. Barrett, Phys. Rev. C57, 562 (1998); P. Navrátil and B.R. Barrett, Phys. Rev. C59, 1906 (1999).

12. P. Navrátil, G.P. Kamuntavičius, and B.R. Barrett, Phys. Rev. C 61, 044001 (1998).

13. J.H. Wilkinson, The Algebraic Eigenvalue Problem, (Clarendon Press, Oxford, 1965).

14. R. Machleidt, F. Sammarruca and Y. Song, Phys. Rev. C53, 1483 (1996).

15. P. Navrátil, J.P. Vary, and B.R. Barrett, Phys. Rev. Lett. 84, 5728 (2000); P. Navrátil, J.P. Vary, and B.R. Barrett, Phys. Rev. C62, 054311 (2000).

16. P. Navrátil, J.P. Vary, W.E. Ormand, and B.R. Barrett, Phys. Rev. Lett. 87, 172501 (2001).

17. E. Caurier, P. Navrátil, W.E. Ormand, and J.P. Vary, Phys. Rev. C64, 051301 (2001)

18. E. Caurier, P. Navrátil, W.E. Ormand, and J.P. Vary, Phys. Rev. C66, 024314 (2002).

19. P. Navrátil and W.E. Ormand Phys. Rev. Lett. 88, 152502 (2002)

20. S.A. Coon, M.D. Scadron, P.C. Mcname, B.R. Barrett, D.W.E. Blatt, B.M.J. McKellar, Nucl. Phys. A317, 242 (1979). 